\title{
Why are influenza vaccination rates low and Tdap vaccination rates average in a large military treatment facility? Survey results from providers and patients
}

\author{
Heather KJinks ${ }^{1}$, Megan E Pagan ${ }^{2}$, Leighann Black ${ }^{1}$, Ashley Adams ${ }^{3}$, Torie Plowden ${ }^{1}$, Everett F Magann ${ }^{2}$ and Karen L Wilson ${ }^{4}$ \\ ${ }^{1}$ Womack Army Medical Center, Department of Obstetrics and Gynecology, 2817 Reilly Road, Fort Bragg, NC 28307, USA \\ ${ }^{2}$ University of Arkansas for Medical Sciences, Department of Obstetrics and Gynecology, Division of Maternal Fetal Medicine, 4301 W. Markham Street, Little \\ Rock, AR 72202, USA \\ ${ }^{3}$ Womack Army Medical Center, Department of Research, 2817 Reilly Road, Fort Bragg, NC 28307, USA \\ ${ }^{4}$ Womack Army Medical Center, Department of Obstetrics and Gynecology, Division of Maternal Fetal Medicine, 2817 Reilly Road, Fort Bragg, NC 28307, USA
}

\begin{abstract}
Objective: The study objective was to determine why vaccination rates in a Military Treatment Facility, where patients have ready access to care at no cost, differ from the general population.

Methods: Influenza and Tdap vaccination rates for pregnant women were analyzed at Womack Army Medical Center from October 1, 2015 to September 30, 2018. Provider and patient surveys were conducted to evaluate barriers. All analyses were descriptive and conducted using SAS 9.4 (SAS Institute, Cary NC) or SPSS 25 (IBM Corp, Armock NY).

Results: 9,448 pregnancies were included. The influenza vaccination rate was $36.7 \%$ and $56.4 \%$ for Tdap. Providers reported offering vaccinations at $>90 \%$ of visits, whereas only $60 \%$ of patients reported being offered vaccination. $73.4 \%$ and $64.4 \%$ of patients declining vaccination reported the risks and benefits were not discussed for influenza and Tdap, respectively. Patients reported "provider recommendation" was the most important factor when choosing to receive vaccinations.

Conclusion: Our influenza vaccination rate was lower and Tdap rate was average compared to the general population. The surveys suggest that provider and patient communication is a significant barrier to vaccination during pregnancy. Providers lending strong support to vaccinations during pregnancy and taking time to address concerns may be key to improving vaccination rates.
\end{abstract}

\section{Introduction}

The Advisory Committee on Immunization Practices (ACIP) at the Center for Disease Control and Prevention (CDC), the American College of Obstetricians and Gynecologists (ACOG), the World Health Organization (WHO) recommends pregnant women or women who will become pregnant receive the most recent influenza virus vaccine at any time during or prior to their pregnancy $[1,2]$. Additionally, it is recommended that pregnant women in the third trimester of pregnancy receive the tetanus, diphtheria, acellular pertussis (Tdap) vaccine, in each pregnancy between 27 and 36 weeks, regardless of prior vaccination status. These vaccinations, when received during pregnancy, provide passive immunity to infants during the first year of life $[1,3]$.

Pregnant women and their infants are particularly vulnerable to the severe complications of infections during pregnancy, to include the seasonal influenza virus, due to their relatively immunocompromised state [4]. Women and infants who are vaccinated with the seasonal influenza vaccine have less severe disease if infected. Infants cannot receive the influenza vaccine until 6 months, however can be provided passive immunity by vaccinated mothers via placental transfer of antibodies conferring immunity until they can be vaccinated $[1,3,5]$. It is estimated the seasonal influenza vaccine has a $63 \%$ efficacy in newborns up to 6 months of age when mothers are vaccinated in pregnancy and the number of febrile, respiratory illness is reduced by $36 \%[5-7]$.

The Tdap vaccine is recommended in each pregnancy regardless of prior vaccination status, due to the high morbidity and mortality of pertussis infection in the neonate within the first year of life [8]. Similar to the influenza vaccination, fetuses receive passive immunity to Bordetella pertussis infection from mother who are vaccinated during pregnancy. Adults are carriers of pertussis and IgG wanes significantly by 1 year after vaccination, necessitating re-vaccination in each pregnancy. Vaccination performed during the third trimester maximizes the passive immunity conferred to the fetus [8]. Vaccinating the contacts of a neonate or pregnant woman, known as cocooning, to prevent whooping cough in the neonate is not an effective vaccination

${ }^{*}$ Correspondence to: Megan Pagan, MD, University of Arkansas for Medical Sciences, Department of Obstetrics and Gynecology, Division of Maternal Fetal Medicine, 4301 W. Markham Street, Little Rock, AR 72202, USA, E-mail: mpagan@uams.edu

Key words: vaccination, pregnancy, influenza, Tdap, vaccination barriers

Received: June 15, 2020; Accepted: November 13, 2020; Published: November 20, 2020 

patients

strategy $[3,8]$. These findings were the catalyst for the ACIP to announce their recommendation for Tdap vaccination in each pregnancy in October 2012.

Despite recommendations from multiple health organizations, the vaccination rates are still far below the Healthy People 2020 goals. Well documented barriers to flu and Tdap vaccination during pregnancy include provider's recommendations, access to care, concern for safety and/or efficacy and cost $[9,10]$. The purpose of this study was to determine the why the vaccination rates were low for influenza and only slightly above average for Tdap in a large Military Treatment Facility (MTF) where there is ready access to care at no cost.

\section{Methods}

\section{Vaccination rate data collection}

A search of the electronic medical record (EMR) was performed and patients who had a prenatal visit after the first trimester at WAMC between October 1, 2015 and September 30, 2018 were identified. Patients were considered to have appropriately received the influenza vaccination if the date of vaccination was within the primary influenza season. If the patient had a pregnancy that spanned over two influenza seasons, then two influenza vaccinations were sought. Patients were considered to have appropriately received the Tdap vaccination if the vaccination date was between the 26 th and 42 nd week of pregnancy.

To determine the influenza or Tdap vaccination rates during pregnancy, some data manipulation was necessary. The EMR used by MTFs does not allow for immunizations to be tracked as received or missing by patient profile, or by estimated due date (EDD) in data reports without an individual chart review. To calculate the rates of vaccinations during pregnancy, three different sets of data were pulled over the time frame October 1, 2015 to September 30, 2018: all of visits with a corresponding ICD-9 code for prenatal visits, all Tdap vaccinations given determined by CPT code and all influenza vaccinations given determined by $\mathrm{CPT}$ code.

The ICD-9 codes for the specific week of pregnancy were used to estimate a due date for the patient. In the case of a single, full-term pregnancy, with no re-dating and no ICD coding errors, the calculated due dates (CDD) were all within 7 days of each other. Given the large number of patients, it was not feasible to assess each of the individual patient records. Therefore, the earliest CDD was taken as the EDD if all CDDs based on the prenatal visits were within 7 days of each other. If there were CDDs 1 to 4 weeks past the earliest CDD, then the latest CDD was accepted as the EDD. Any CDDs that were more than 40 weeks beyond the earliest CDD were considered a separate pregnancy.

Less than $5 \%$ of the data were patients with CDDs 8 to 40 weeks apart and were excluded from the analysis. Patients were also excluded if there was no week-specific data for any prenatal visit or if there were no visits beyond the first trimester. The remaining patients were separated by their individual pregnancies and their EDD, and were cross referenced with all Tdap and flu vaccinations given during the same timeframe. For each pregnancy, we were able to determine if a Tdap vaccination had been given between 14 weeks prior and 2 weeks after the EDD, or from the 26th to 42nd week of the pregnancy. For each pregnancy, it was determined if the influenza vaccine(s) were given during the applicable influenza season(s).

\section{Patient questionnaire}

The patient questionnaire consisted of 23 questions and included questions regarding patient demographics, pregnancy, vaccination choices and vaccination beliefs. The survey was distributed to patients during the month of April 2019 in the obstetrics clinic and contained no personally identifiable information. The survey tool was specific to the MTF and not rated for reliability or validity.

\section{Provider questionnaire}

The provider questionnaire was conducted online and consisted of 10 items with questions regarding vaccination recommendation practices and providers' perceptions of patient's attitude or beliefs regarding vaccines during the months of April and May in 2018. The survey link was sent via email to obstetric health care providers during the study period. The questions, which were specific to the MTF, were validated by the study staff but not rated for reliability.

\section{Statistical methods}

Categorical variables were reported in counts and frequencies. All analyses were descriptive and conducted using SAS 9.4 (SAS Institute, Cary NC) or SPSS 25 (IBM Corp, Armock NY).

\section{Results}

\section{Vaccination rates}

The prenatal data set included all documented visits for patients, ranging from one visit to multiple over the course of one or more pregnancies, for a total of $\mathrm{N}=92,837$ visits associated with 10,868 patients. Patients with indeterminate EDD (difference of 4-8 weeks in confirmed delivery dates (CDD)s, $\mathrm{N}=541$ ), no week-specific ICD codes $(\mathrm{N}=261)$ and those with no visits beyond the first trimester $(\mathrm{N}=1,545)$ were excluded. Patients included in the study consisted of $\mathrm{N}=8,521$ patients with $\mathrm{N}=9,448$ pregnancies.

Active duty and dependent patients had higher Tdap vaccination rates during the study period (59.4\% and $56.7 \%$, respectively) than the 2016-2017 national average (50.0\%). Patients classified as "Others", those who fit neither category (such as retired servicemembers), had a lower Tdap vaccination rate $(40.9 \%$, Figure 1 .)

The specific rate of active duty patients who received the influenza vaccination was unable to be calculated because service members receive mandatory vaccination as part of their unit. This information is not recorded by CPT code or in a reportable field. An audit of 20 randomly selected charts suggested $95 \%$ compliance in active duty patients. Dependent and Other patients had lower rates of influenza vaccination than the $2016-2017$ national average (36.7\% and $19.6 \%$ vs. $54.0 \%$, Figure 1).

\section{Patient questionnaire}

During April 2019, 327 patients completed the written patient questionnaire. Most patients were not active duty service members (78.9\%) and had been to the OB/GYN clinic previously (85.6\%, Table 1). There was distribution across all gestational ages at the time the survey was distributed: $9.5 \%$ were $5-12$ weeks, $27.8 \%$ were $13-24$ weeks, $22.2 \%$ were $25-32$ weeks, and $30.9 \%$ were $32+$ weeks pregnant.

Most patients (92.7\%) did not report going to another clinic in the hospital to receive immunizations was a vaccination barrier. More than two-thirds of patients reported receiving the influenza vaccination during the 2018-2019 flu season (Table 2). 60.6\% active duty service members reported their unit requires them to get the flu vaccine and $21.2 \%$ reported their unit directed them to their Obstetrician for vaccination. Over half of patients $(60.9 \%)$ recalled their provider recommending the influenza vaccine while $32.1 \%$ reported not being 

patients

offered vaccination; $76.7 \%$ reported receiving the vaccine after their provider's recommendation. Of patients who declined the vaccination, $73.4 \%$ reported that the risks and benefits were discussed with them. When asked the purpose of receiving the influenza vaccine, $63.3 \%$ knew that the vaccine served to both prevent influenza and to lessen the severity of illness (Table 2). A further $31.1 \%$ knew at least one benefit of the vaccine. The majority of patients reported that the vaccine did not cause either influenza (86.2\%) or birth defects/Autism (84.1\%).

Approximately $40 \%$ of patients reported receiving the Tdap vaccination, although $13.2 \%$ noted that they less than 28 weeks pregnant and not yet eligible to receive the vaccine (Table 2). However, in a later question, $48.0 \%$ of patients said that they were not given a recommendation for the vaccine as it was not yet applicable. Slightly fewer patients recalled receiving a recommendation for the Tdap vaccine from their provider $(42.2 \%)$, and $83.8 \%$ of patients reported they had or would receive the vaccine. Of patients who declined Tdap vaccination, $64.4 \%$ reported being counseled on the risks and benefits. When asked the purpose of receiving the Tdap vaccine, $52.0 \%$ answered 'To provide passive immunity to the baby after it's born', while $29.4 \%$ reported they did not know the purpose of the vaccine. The majority of patients $(80.7 \%)$ believed that the Tdap vaccine does not cause birth defects/autism.

When asked about their influences when considering vaccination, nearly $80 \%$ ranked their Obstetrics provider as their primary source of information (Figure 2), with family/friends and other healthcare providers ranking similarly as secondary sources of information.

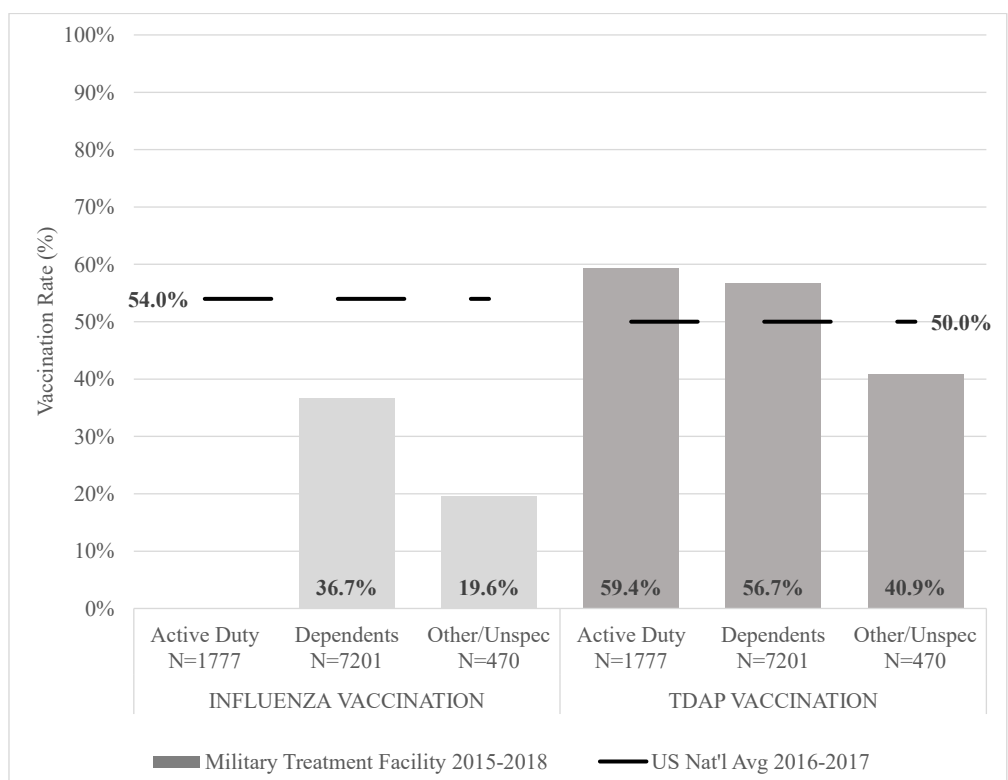

Figure 1. Vaccination Rates in Pregnancy at a Military Treatment Facility as Compared to US National Averages. The rate of active duty patients who received the influenza vaccination was unable to be calculated; nearly all service members receive mandatory vaccination as part of their unit

Table 1. Participant characteristics

\begin{tabular}{|c|c|c|}
\hline Patient Characteristics & & $\mathbf{N}=\mathbf{3 2 7}$ \\
\hline \multicolumn{3}{|c|}{ How many weeks pregnant are you? } \\
\hline & 5-12 Weeks & $31(9.5)$ \\
\hline & 13-24 Weeks & $91(27.8)$ \\
\hline & 25-32 Weeks & $72(22.2)$ \\
\hline & $32+$ Weeks & $101(30.9)$ \\
\hline & Post-partum & $26(8.0)$ \\
\hline & Not pregnant & $5(1.5)$ \\
\hline & Not answered & $1(0.3)$ \\
\hline \multicolumn{3}{|l|}{ Are you active duty? } \\
\hline & Yes & $67(20.5)$ \\
\hline & No & $258(78.9)$ \\
\hline & Other (Reserves/National Guard) & $2(0.6)$ \\
\hline \multicolumn{3}{|c|}{ Is this your first appointment at this clinic? } \\
\hline & Yes & $43(13.2)$ \\
\hline & No & $280(85.6)$ \\
\hline & Other (Post-partum/not pregnant) & $4(1.2)$ \\
\hline \multicolumn{3}{|c|}{ Do you think having to go upstairs to the Allergy/Immunology clinic is a barrier to getting either vaccine? } \\
\hline & Yes & $20(6.1)$ \\
\hline & No & $303(92.7)$ \\
\hline & Other (Sometimes, not answered, etc.) & $4(1.2)$ \\
\hline
\end{tabular}

Data reported in $\mathrm{N}(\%)$. 
Jinks HK (2020) Why are influenza vaccination rates low and Tdap vaccination rates average in a large military treatment facility? Survey results from providers and patients

Table 2. Patient responses regarding influenza and Tdap vaccinations

Patient Responses to Vaccination Questions (N=327)

Have you received the vaccine (during this flu season?/during this pregnancy?)

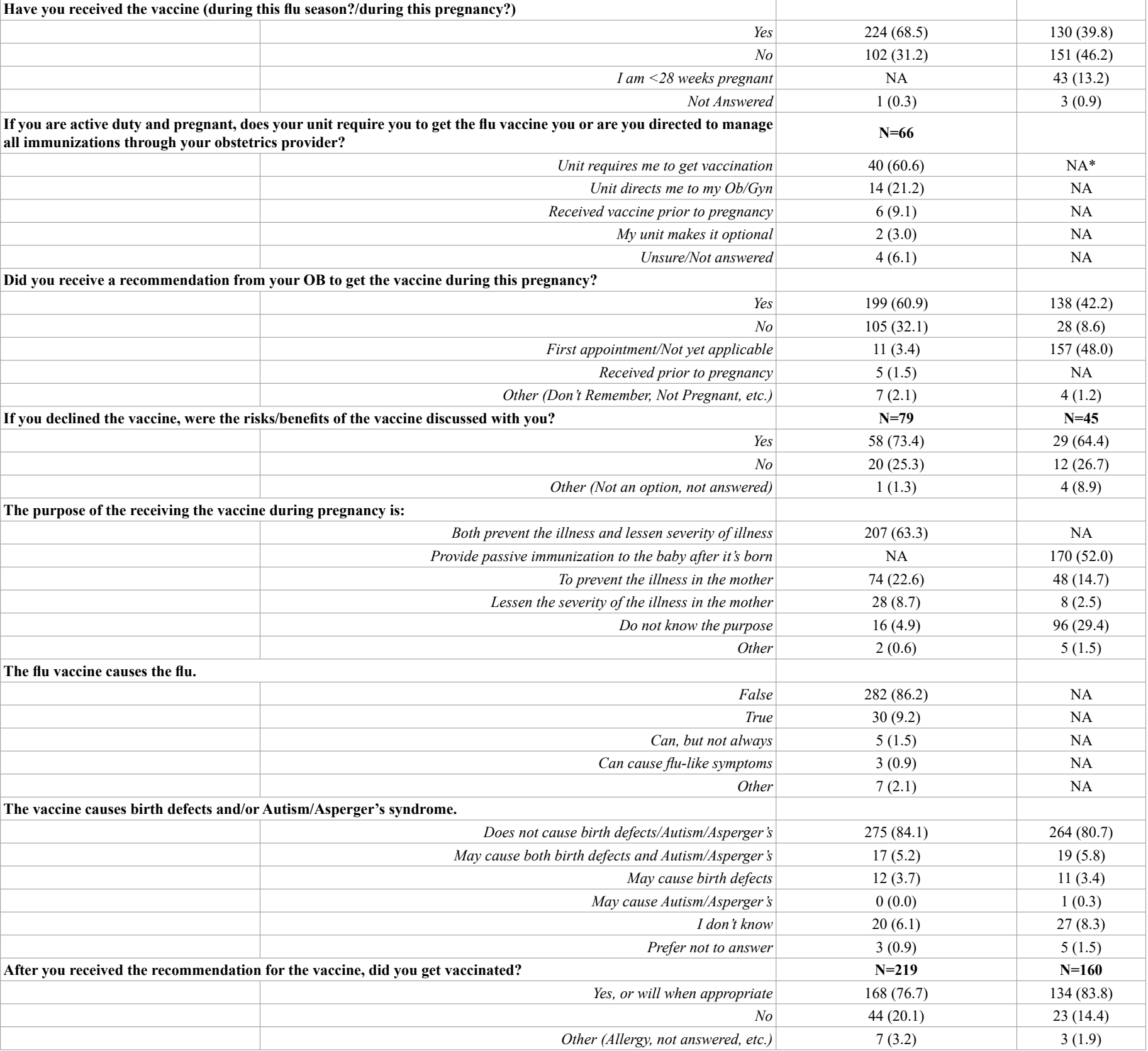

Data reported in N (\%). *NA - Not Asked.

\section{Provider questionnaire}

During the two-month provider questionnaire time frame, 51 providers responded. The majority reported always offering the influenza (90.2\%) and Tdap (94.1\%) vaccines to their pregnant patients (Table 3). Over $80 \%$ of providers were not at all concerned about either immunization in regard to maternal safety, fetal safety or vaccine contents. Providers were not at all concerned about the effectiveness of the Tdap vaccine ( $88.2 \%)$, but almost half were at least mildly concerned about the effectiveness of the influenza vaccine (43.1\%).
When vaccination is offered, over $90 \%$ of providers report discussing the risks and benefits with the patient (Table 3 ). Approximately half will discuss the risks and benefits if the patient initially refuses vaccination or if the patient asks about the risks and benefits. Providers who do not have vaccinations available in their clinic believe that this is a barrier $(88.9 \%)$ and believe patients perceive this as a barrier (72.2\%).

Providers report that the reasons patients 'most often' or 'somewhat often' refuse vaccinations are because they are uneducated regarding 
Jinks HK (2020) Why are influenza vaccination rates low and Tdap vaccination rates average in a large military treatment facility? Survey results from providers and patients

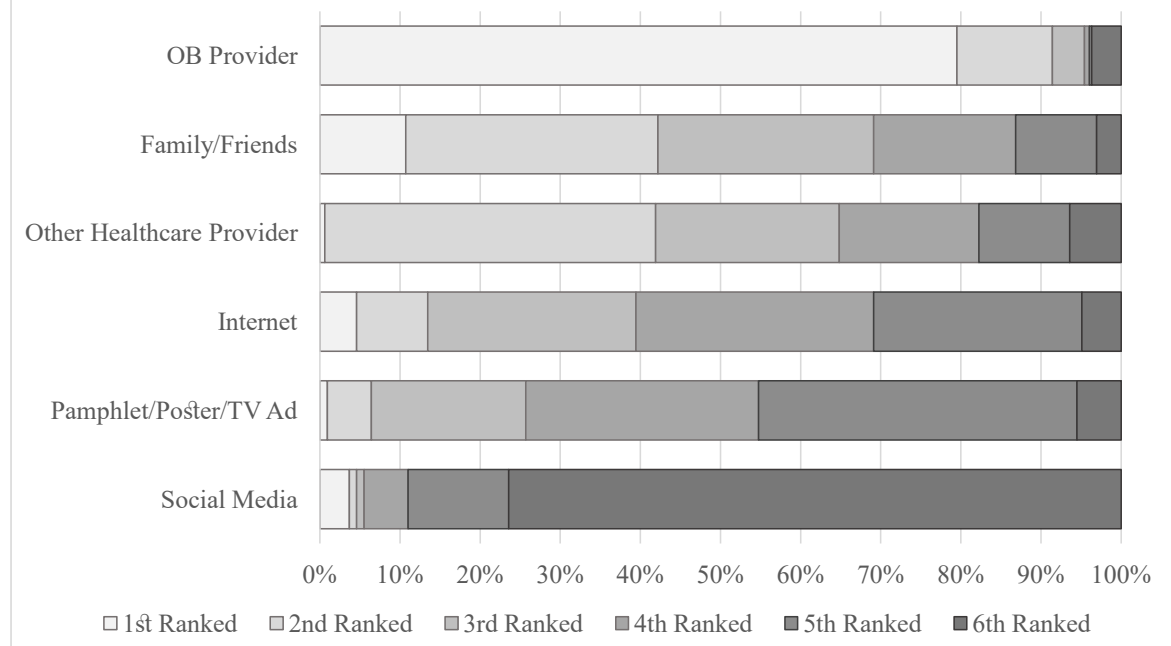

Figure 2. Ranking of Vaccination Influences. Ranked responses to the question "Of the many sources of information, where one(s) do you consider the most influential when considering getting the flu vaccine during pregnancy?"

Table 3. Provider responses regarding influenza and Tdap vaccinations

Provider Responses to Vaccination Questions ( $\mathbf{N}=\mathbf{5 1}$ )

How often do you offer the vaccine to patients during their pregnancy?

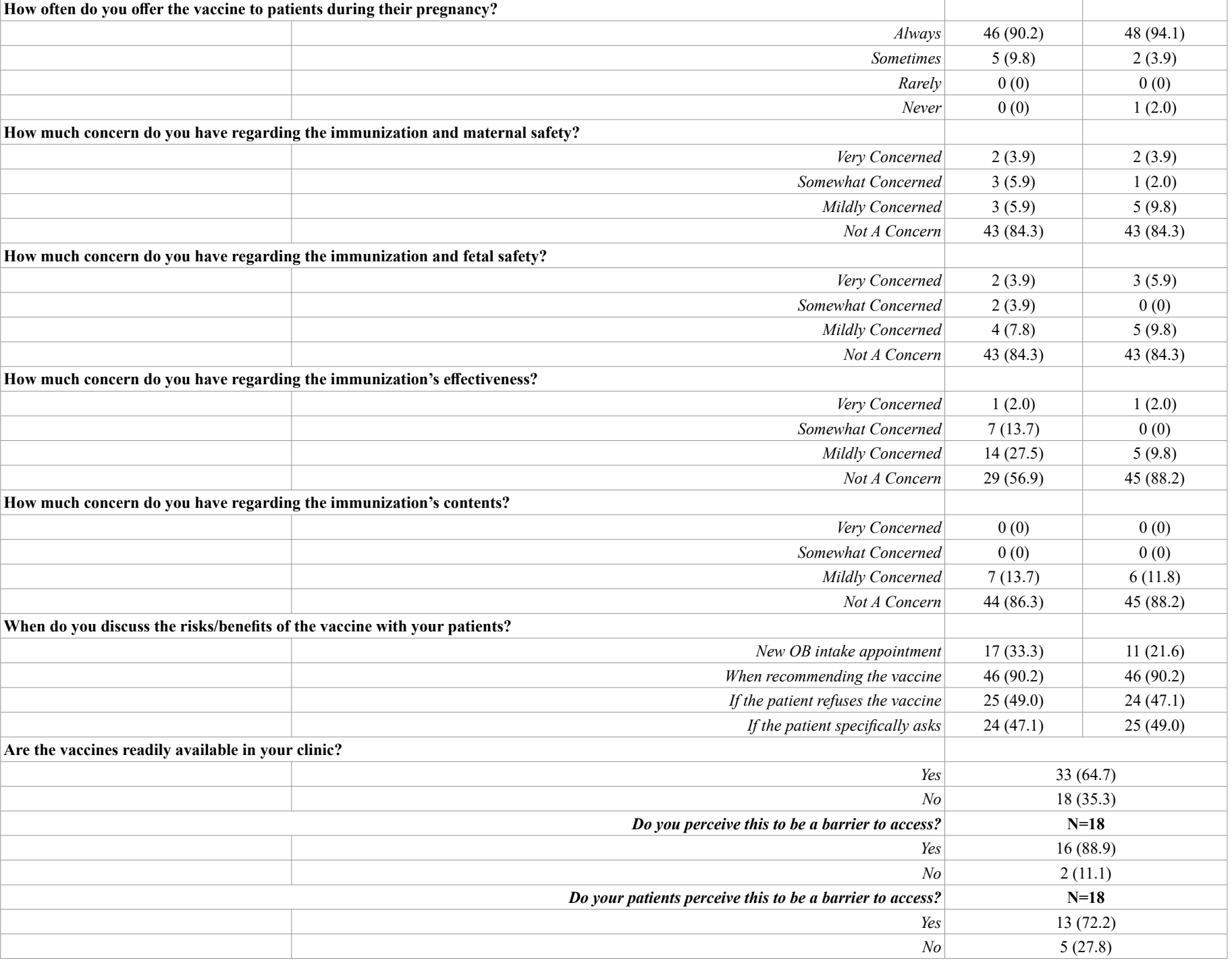

Data reported in N (\%). 
Jinks HK (2020) Why are influenza vaccination rates low and Tdap vaccination rates average in a large military treatment facility? Survey results from providers and patients

risks of the influenza $(68.7 \%)$ or pertussis infection $(56.9 \%$, Figure 3 , Table 4). In providers' experiences, patients 'often' or 'somewhat often' refuse the influenza vaccine because they believe it causes the flu (60.8\%), but this is 'not often' or 'somewhat often' a cause for Tdap refusals (15.7\%). Providers report that when the vaccines are refused, approximately a fifth of patients 'often' or 'somewhat often' mention beliefs that the influenza (23.5\%) or Tdap (21.6\%) vaccine causes birth defects or other cognitive deficits.

\section{Discussion}

Among pregnant women who received prenatal care at WAMC, influenza vaccination coverage lower than the general population vaccination rate, however Tdap vaccination rates were higher than the general population. ${ }^{11}$ The reasons for the low vaccination rate for the flu is uncertain. This study does provide information that may help us to increase the vaccination rate in our obstetric patient population.

When providers were asked how often patients mention the belief that the vaccines cause birth defects or autism, 52.9\% responded 'rarely' or 'never' for the flu vaccine and 56.9\% ('rarely' or 'never' for the Tdap vaccine. This information was dissimilar to the providers' survey where they reported that, $84.1 \%$ and $80.7 \%$ of patients reported that the flu and Tdap vaccine causes birth defects or autism. This suggests that although the anti-vaccine movement is prominent in the media and in social media, the majority of our patients do not believe that the vaccine causes either.

Our provider survey showed that over $90 \%$ of providers are offering these vaccinations to their pregnant patients however the patient survey showed that only $60 \%$ of patients report flu vaccination being recommended by their provider. In a recent study involving provider surveys regarding administration of antepartum Tdap vaccination, $11 \%$ of providers reported not routinely offering the Tdap vaccine to pregnant patients and $16 \%$ reported that they deferred the administration of the vaccinations to primary care physician. These findings suggest education regarding vaccinations in pregnancy is needed for both providers and patients.

Recently, the CDC examined early influenza vaccination rates in pregnancy from July 2017 to November 2017 [12]. Of those women who had seen their physician, only $58.7 \%$ received a recommendation

Table 4. Provider responses regarding patient refusal of influenza and Tdap vaccinations

\begin{tabular}{|c|c|c|c|}
\hline \multicolumn{2}{|l|}{ Provider Perspectives of Patient Beliefs $(\mathrm{N}=51)$} & Influenza & Tdap \\
\hline \multicolumn{4}{|c|}{ When your patient declines vaccinations, how often do they mention: } \\
\hline \multicolumn{4}{|l|}{ Concerns raised in online research? } \\
\hline & Often & $9(17.7)$ & $5(9.8)$ \\
\hline & Somewhat Often & $9(17.7)$ & $6(11.8)$ \\
\hline & Occasionally & $18(35.3)$ & $13(25.5)$ \\
\hline & Rarely or Never & $15(29.4)$ & $27(52.9)$ \\
\hline \multicolumn{4}{|l|}{ Concerns raised by family or friends? } \\
\hline & Often & $6(11.8)$ & $4(7.8)$ \\
\hline & Somewhat Often & $13(25.5)$ & $8(15.7)$ \\
\hline & Occasionally & $14(27.5)$ & $12(23.5)$ \\
\hline & Rarely or Never & $18(35.3)$ & $27(52.9)$ \\
\hline \multicolumn{4}{|l|}{ Allergy or prior vaccine reaction? } \\
\hline & Often & $2(3.9)$ & $3(5.9)$ \\
\hline & Somewhat Often & $12(23.5)$ & $1(2.0)$ \\
\hline & Occasionally & $20(39.2)$ & $17(33.3)$ \\
\hline & Rarely or Never & $17(33.3)$ & $30(58.8)$ \\
\hline \multicolumn{4}{|l|}{ Uneducated about the risk of specific diseases? } \\
\hline & Often & $19(37.3)$ & $11(21.6)$ \\
\hline & Somewhat Often & $16(31.4)$ & $18(35.3)$ \\
\hline & Occasionally & $9(17.7)$ & $10(19.6)$ \\
\hline & Rarely or Never & $7(13.7)$ & $12(23.5)$ \\
\hline \multicolumn{4}{|c|}{ Believes vaccines causes birth defects and/or cognitive deficits? } \\
\hline & Often & $7(13.7)$ & $6(11.8)$ \\
\hline & Somewhat Often & $5(9.8)$ & $5(9.8)$ \\
\hline & Occasionally & $12(23.5)$ & $11(21.6)$ \\
\hline & Rarely or Never & $27(52.9)$ & $29(56.9)$ \\
\hline \multicolumn{4}{|l|}{ Believes vaccine cases the disease? } \\
\hline & Often & $17(33.3)$ & $5(9.8)$ \\
\hline & Somewhat Often & $14(27.5)$ & $3(5.9)$ \\
\hline & Occasionally & $11(21.6)$ & $4(7.8)$ \\
\hline & Rarely or Never & $9(17.7)$ & $39(76.5)$ \\
\hline \multicolumn{4}{|l|}{\begin{tabular}{|l|} 
No explanation? \\
\end{tabular}} \\
\hline & Often & $5(9.8)$ & $7(13.7)$ \\
\hline & Somewhat Often & $13(25.5)$ & $11(21.6)$ \\
\hline & Occasionally & $20(39.2)$ & $11(21.6)$ \\
\hline & Rarely or Never & $13(25.5)$ & $22(43.1)$ \\
\hline
\end{tabular}

Data reported in $\mathrm{N}(\%)$. 


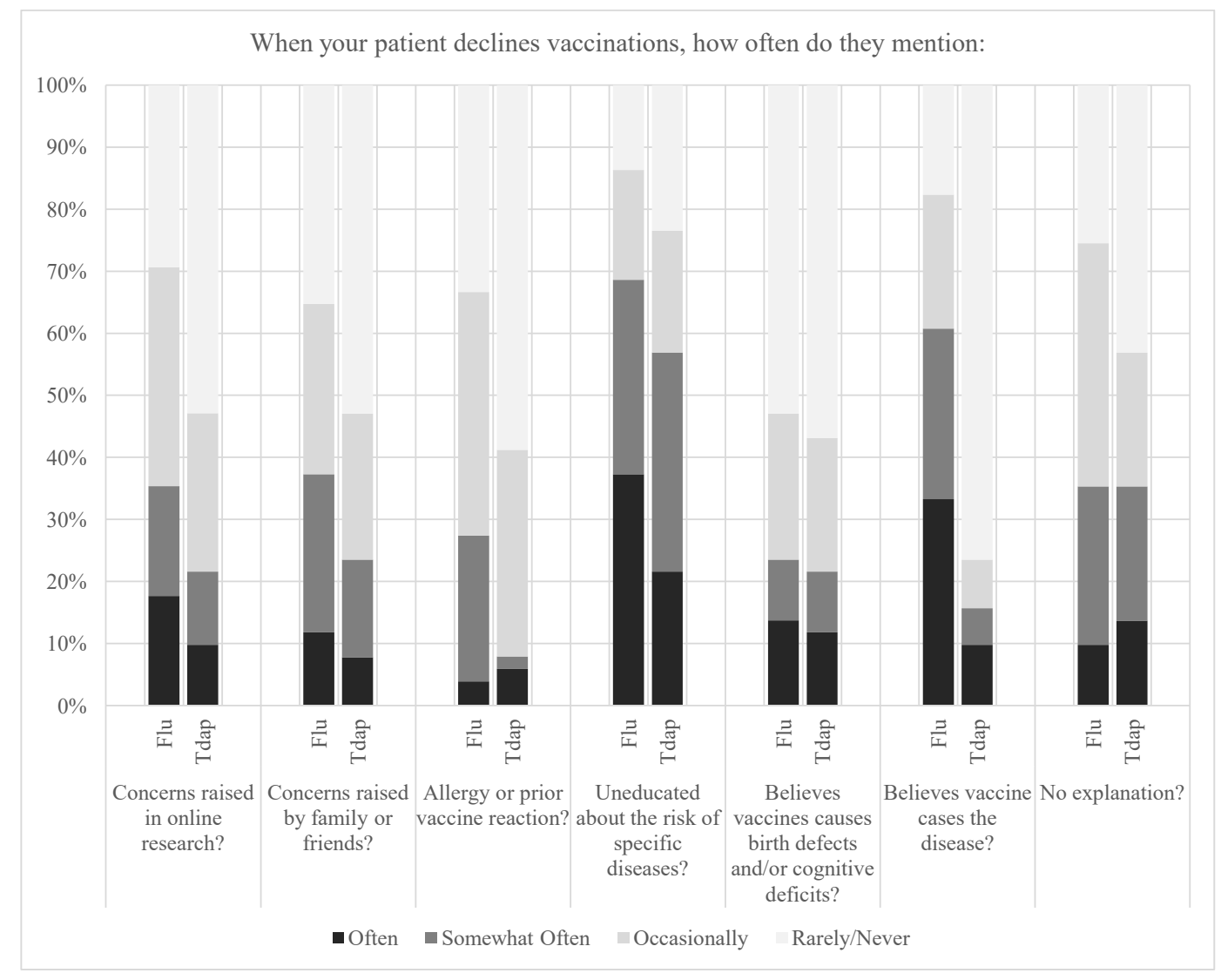

Figure 3. Provider perceptions regarding patient refusal of influenza and Tdap vaccinations

and offer for vaccination and $25.7 \%$ reported receiving neither a recommendation nor an offer to receive the influenza vaccine. Among those women, influenza vaccination rates were $52.4 \%$ and $5.7 \%$ respectively. These findings support the result of our study that a providers' recommendation for vaccination is a significant factor in a patient receiving a vaccine and therefore, has a significant impact on vaccination rates. Interestingly, $90 \%$ of providers reported offering the influenza vaccine however, only $60 \%$ of patients reported receiving a recommendation for the vaccine. This suggests that what the patient interprets the provider says and what a provider says has a significant disconnect.

The principle strength of this study is the large sample size of women of women treated at this MTF. Limitations to this study include the use of invalidated patient and provider surveys as well as some questions from the surveys being excluded as they were too vague and were unclear. We could not obtain a vaccination rate for active duty servicemembers due to the manner in which they are recorded in the EMR however, a random chart review suggested a high vaccination rate which would be expected as the flu vaccine is required for all active duty servicemembers.

We have the opportunity to reduce maternal and neonatal morbidity and mortality with these vaccination, yet despite the unanimous recommendations for influenza and Tdap vaccination during pregnancy from health agencies around the world, the vaccination rates in the U.S. remains at approximately 50\% [11]. The CDC's Healthy People 2020 goals for influenza and Tdap vaccination rates during pregnancy are $80 \%$ and $70 \%$, respectively [13-15]. The U.S. is currently far below the goals for both vaccinations and barriers to vaccination need to be addressed for us to be able to meet this goal. Providers lending strong support to vaccinations during pregnancy and taking time to address concerns regarding the safety and purpose of vaccinations multiple times during the pregnancy (even at each visit ) during pregnancy may be a key factor to improving vaccination rates.

\section{Financial disclosure}

No funding or financial support was received for this work.

\section{Disclaimer}

The opinions or assertions contained herein are the private views of the authors and are not to be construed as the official policy of the Department of the Army, Department of Defense, or the U.S. Government.

\section{References}

1. ACOG Committee Opinion No. 741: Maternal Immunization. Obstet Gynecol. 2018. 131: e214-e217.

2. ACOG Committee Opinion No. 732 Summary: Influenza Vaccination During Pregnancy. Obstet Gynecol. 2018. 131: 752-753.

3. Healy CM, Rench MA, Wootton SH, Castagnini LA (2015) Evaluation of the impact of a pertussis cocooning program on infant pertussis infection. Pediatr Infect Dis $J$ 34: $22-26$.

4. Grohskopf LA, Sokolow LZ, Broder KR, Walter EB, Fry AM, et al. (2018) Prevention and control of seasonal influenza with vaccines: Recommendations of the advisory committee on immunization practices-United States, 2018-19 Influenza Season. MMWR Recomm Rep 67: 1-20.

5. Zaman K, Roy E, Arifeen SE, Raqib R, Wilson E, et al. (2008) Effectiveness of maternal influenza immunization in mothers and infants. $N$ Engl J Med 359: 15551564. [Crossref] 

patients

6. Tapia MD, Sow SO, Tamboura B, Tégueté I, Pasetti MF, et al. (2016) Maternal immunisation with trivalent inactivated influenza vaccine for prevention of influenza in infants in Mali: a prospective, active-controlled, observer-blind, randomised phase 4 trial. Lancet Infect Dis 16: 1026-1035. [Crossref]

7. Steinhoff MC, Katz J, Englund JA, Khatry SK, Shrestha L, et al. (2017) Year-round influenza immunisation during pregnancy in Nepal: a phase 4, randomised, placebocontrolled trial. Lancet Infect Dis 17: 981-989. [Crossref]

8. Chiappini E, Stival A, Galli L, de Martino M (2013) Pertussis re-emergence in the postvaccination era. BMC Infect Dis 13: 151. [Crossref]

9. MacDougall DM, Halperin SA (2016) Improving rates of maternal immunization: Challenges and opportunities. Hum Vaccin Immunother 12: 857-865. [Crossref]

10. O'Leary ST, Riley LE, Lindley MC, Allison MA, Albert AP, et al. (2019) ObstetricianGynecologists' strategies to address vaccine refusal among pregnant women. Obstet Gynecol 133: 40-47. [Crossref]
11. Kahn KE, Black CL, Ding H, Williams WW, Lu PJ, et al. (2018) Influenza and Tdap vaccination coverage among pregnant women - United States, April 2018. MMWR Morb Mortal Wkly Rep 67: 1055-1059.

12. Helen DM, Black CL, Ball SW, Fink RV, Fiebelkorn AP (2018) Pregnant Women and Flu Vaccination, Internet Panel Survey, United States, November 2017.

13. Updated recommendations for use of tetanus toxoid, reduced diphtheria toxoid, and acellular pertussis vaccine (Tdap) in pregnant women--Advisory Committee on Immunization Practices (ACIP), 2012. MMWR Morb Mortal Wkly Rep. 2013. 62: 131-135.

14. Seasonal influenza vaccination coverage among women who delivered a live-born infant - 21 states and New York City, 2009-10 and 2010-11 influenza seasons. MMWR Morb Mortal Wkly Rep. 2013. 62: 1001-1004.

15. Barber A, Muscoplat MH, Fedorowicz A (2017) Coverage with Tetanus, Diphtheria, and Acellular Pertussis Vaccine and Influenza Vaccine Among Pregnant Women Minnesota, March 2013-December 2014. MMWR Morb Mortal Wkly Rep 66: 56-59.

Copyright: (C2020 Jinks HK. This is an open-access article distributed under the terms of the Creative Commons Attribution License, which permits unrestricted use, distribution, and reproduction in any medium, provided the original author and source are credited. 